\title{
Ghlorinity/salinity distribution patterns in experimental granular sea ice
}

\author{
J.-L. Tison, V. VERBEKe \\ Laboratoire de Glaciologie, Département des Sciences de la Terre et de l'Environnement, Faculté des Sciences, CP 160/03, \\ Université Libre de Bruxelles, B-1050 Brussels, Belgium
}

\begin{abstract}
This work investigates the possibility of brine-channel formation and development during the freezing of granular ice from a loose frazil-ice suspension in an $\mathrm{NaCl}$ solution at sea-water concentration. Three experiments were performed at various constant growth rates in a purpose-built vessel with computer-controlled thermal driving. High-resolution chlorinity measurements are used as a proxy for the bulk salinity of the samples. These show clear brine-segregation processes in the ice, with very high salinity gradients for the fast $\left(10 \mathrm{~mm} \mathrm{~h}^{-1}\right)$ to medium $\left(2 \mathrm{~mm} \mathrm{~h}^{-1}\right)$ freezing rates, provided that a suitable sampling scale is adopted. Weak segregation was found at the low freezing rate $\left(0.5 \mathrm{~mm} \mathrm{~h}^{-1}\right)$. The spatial distribution of the bulk salinity fits the visual appearance of brine channels in the ice adequately, in both horizontal and vertical sections. In a similar way to columnar-congelation sea ice, the number of brine channels significantly decreases with growth rate, but the density of channels is systematically lower in the granular ice than that found at equivalent freezing rates in the columnar ice. This is attributed to the lower geometrical constraints on brine transport in the granular medium. Contrasts between brine-channel geometry and density at different growth rates are discussed in light of the "mushy-layer" concept adapted to sea-ice growth from the solidification of alloys.
\end{abstract}

\section{BACKGROUND}

The visual patterns of impurity distribution in natural sea ice have long been satisfactorily described, but recent developments in sea-ice biology, sea-ice modelling and remote sensing call for further insights into the detailed formation and evolution processes of these patterns. Among these impurities, salts are dominant. They are included either as pockets of liquid brine within the solid ice matrix or, depending on the temperature, as precipitated salts. The brine inclusions are the main component of sea-ice porosity. They occur at different scales: brine layers between intra-crystalline ice lamellae $(<1 \mathrm{~mm}$ ), occluded brine pockets (a few $\mathrm{mm}$ in size) or sub-vertical brine channels with inclined "feeder" branches that can extend through most of the sea-ice thickness. These various features are more or less interconnected, depending on the temperature field. Brine channels, since they show the largest vertical extension, are the best candidates for control of brine-transport processes and therefore ocean-atmosphere exchange and biological activity.

Models for initial salt entrapment in sea ice and further desalination processes have been refined (Cox and Weeks, 1988; Eicken, 1998) and experimental work has recently focused on the study of brine-channel formation and evolution processes in columnar-congelation sea ice (Wettlaufer and others, 1997a, b; Cottier, 1999; Cottier and others, 1999). Wettlaufer and others (1997a,b) show that brine-channel development in the early stages of freezing can be understood if brine movement in the growing ice cover is considered as an analogue to convection in mushy layers during the solidification of metals.

Although it can form an important percentage of the sea-ice cover, particularly in the Antarctic, granular sea ice has been less frequently investigated in the laboratory. It is known that brine channels which initiate in a columnar layer of a given natural sea-ice core will usually continue

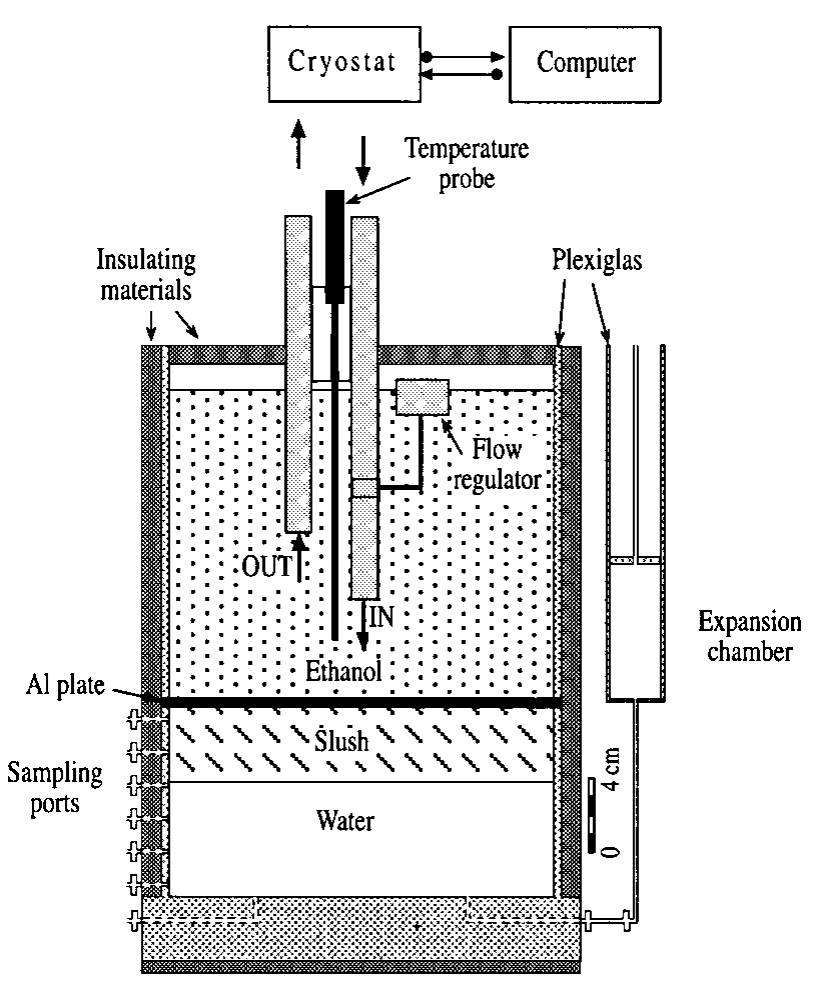

Fig. 1. The computer-controlled freezing apparatus. 
$10 \mathrm{mmh}^{-1}$

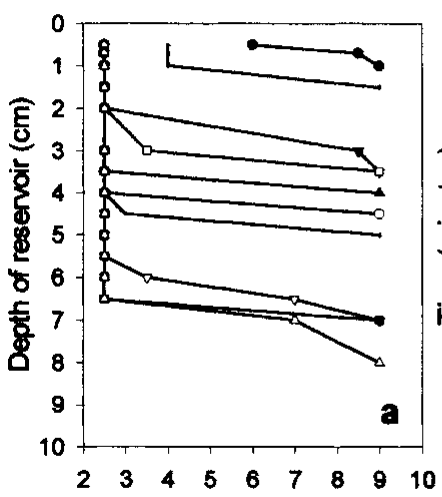

Needle penetration $(\mathrm{cm})$
$10 \mathrm{mmh}^{-1}$

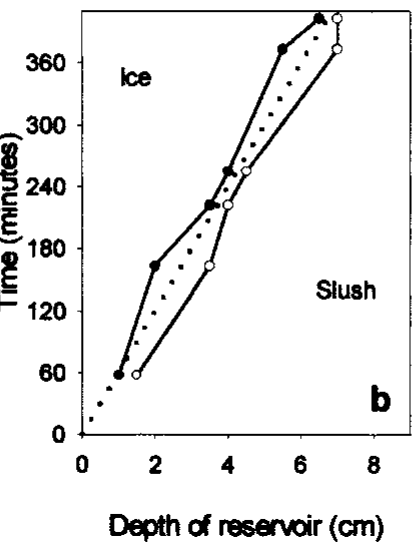

$2 \mathrm{mmh}^{-1}$

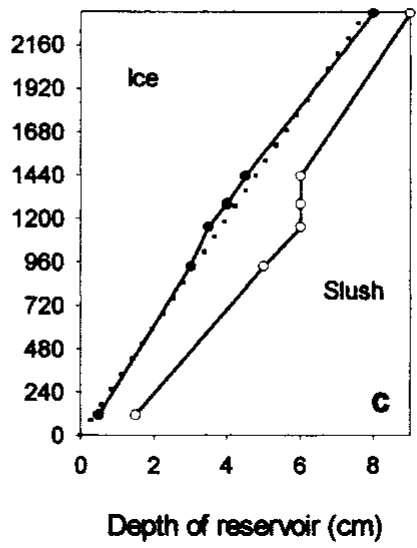

$0.5 \mathrm{mmh}^{-1}$

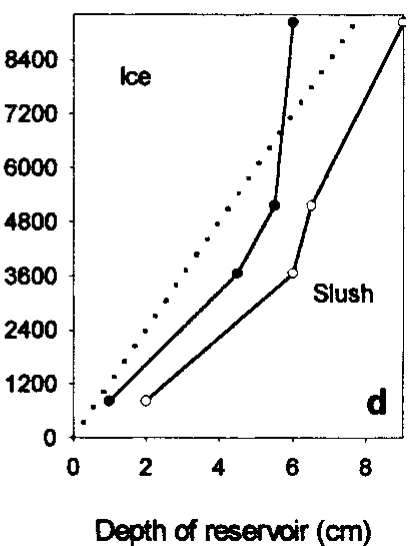

Fig. 2. Control of the downward progression of the freezing front (growth rate) in the freezing apparatus. (a) At a given time and depth, a needle inserted in the sampling ports on the side of the apparatus (depth spacing $0.5 \mathrm{~cm}$ ) shows various degrees of penetration, depending on the consolidation status. Each curve corresponds to a given time (between 67 min (black dots) and 495 min (white triangles)). For example, at $t=440 \mathrm{~min}$ (inverted white triangles), the needle penetrated $3.5 \mathrm{~cm}$ into the slush at $6 \mathrm{~cm}$ depth, but could not get into the ice at $5.5 \mathrm{~cm}$ depth. $(b-d)$ Needle penetration at various depths, as a function of time for the three experiments. Dotted line is the theoretical progression of the freezing front; black dots show depth levels where the needle did not penetrate the ice; and open circles show depth levels where the needle met no resistance in the slush.

$10 \mathrm{mmh}^{-1}$ - row 2

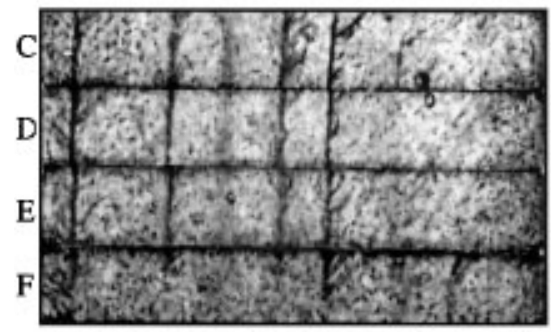

$2 \mathrm{mmh}^{-1}-$ row 7

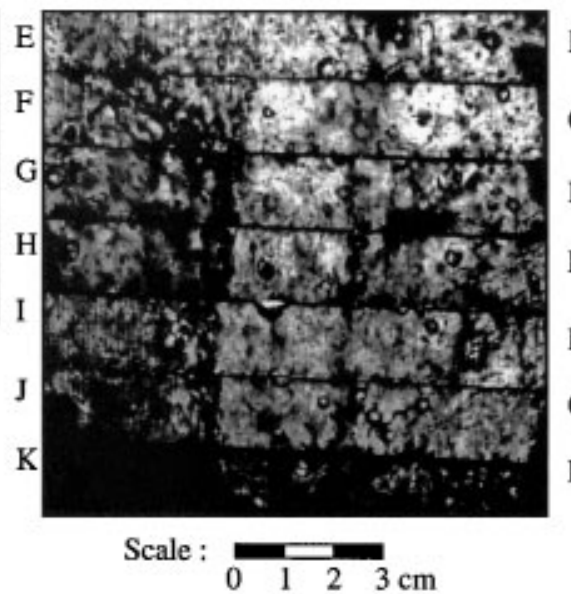

$0.5 \mathrm{mmh}^{-1}$ - row 7

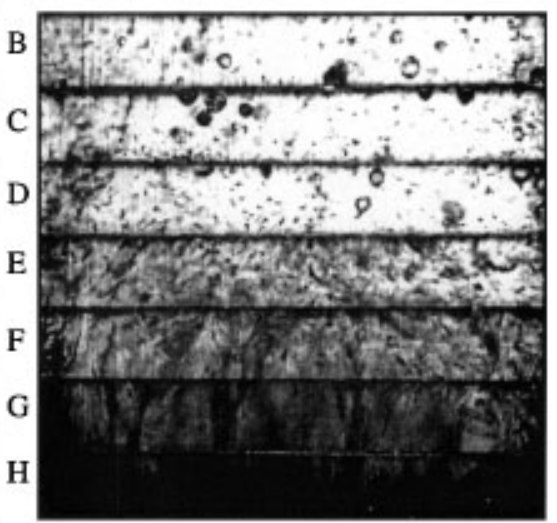

Fig. 3. Typical vertical sections for the three experiments. Capital letters indicate plates ( see Table 1). Congelation ice developed below the slush at the bottom of each experiment (plate $K$ in the $2 \mathrm{~mm} \mathrm{~h}^{-1}$ experiment; plates $\mathrm{F}$-H in the $0.5 \mathrm{~mm} \mathrm{~h}^{-1}$ experiment). Brine channels clearly develop as linear darker sub-vertical features in the 10 and $2 \mathrm{~mm} \mathrm{~h}^{-1}$ experiments. They do not show up in the $0.5 \mathrm{~mm} \mathrm{~h}^{-1}$ section.

down in the granular layer below, if it is present. However, initiation of brine channels in granular sea ice has not been studied per se. Cottier and others (1999) briefly described one experimental run in which granular ice was found to be devoid of any macroscopic features or organized threedimensional salinity field.

This work reports on an experimental study of the possible development of brine channels during consolidation of a loose frazil mixture in an $\mathrm{NaCl}$ solution at sea-water salinity.

\section{EXPERIMENTAL PROCEDURE AND ANALYTICAL TECHNIQUES}

Three experiments of progressive downward freezing at a constant rate (10, 2 and $0.5 \mathrm{~mm} \mathrm{~h}^{-1}$, respectively) were conducted in an insulated purpose-built vessel installed in a cold room at $-2 \pm 2^{\circ} \mathrm{C}$.

The apparatus (Fig. 1) consisted of two cylindrical parts: a lower freezing chamber, $24 \mathrm{~cm}$ in diameter, and an upper alcohol-filled container. The alcohol was systematically cooled to produce a constant growth rate within the lower chamber. The alcohol temperature (calculated by balancing terms for the conduction and the latent heat of fusion at the interface) was adjusted by computer control attached to a Julabo FT50 cryostat.

Individual frazil-ice crystals were generated by placing a $65 \mathrm{~L}$ open bucket filled with $\mathrm{NaCl}$ solution at about $34 \%$ (actually $33.7-33.97 \%$ ) to cool down in a $-25^{\circ} \mathrm{C}$ cold room. A forced ventilation system with a directed flow was used to 
Table 1. Sampling details for the three experimental blocks

\begin{tabular}{|c|c|c|c|}
\hline $\begin{array}{l}10 \mathrm{~mm} \mathrm{~h}^{-1} \\
\text { ow resolution) }\end{array}$ & $\begin{array}{c}10 \mathrm{~mm} \mathrm{~h}^{-1} \\
\text { (high resolution) }\end{array}$ & $2 m m h^{-1}$ & $0.5 m m h^{-1}$ \\
\hline
\end{tabular}

$\begin{array}{lcccc}\text { Block width 1 } & 10 \mathrm{~cm} & 4 \mathrm{~cm} & 10 \mathrm{~cm} & 10 \mathrm{~cm} \\ \text { Block width 2 } & 10 \mathrm{~cm} & 3 \mathrm{~cm} & 10 \mathrm{~cm} & 10 \mathrm{~cm} \\ \text { Block depth } & 8.5 \mathrm{~cm} & 8.5 \mathrm{~cm} & 10.5 \mathrm{~cm} & 11 \mathrm{~cm} \\ \text { Number of plates } & 7 & 7 & 11 & 8 \\ \text { Plate thickness } & 1.2 \mathrm{~cm} & 1.2 \mathrm{~cm} & 0.45 / 1.2 \mathrm{~cm} & 1.35 \mathrm{~cm} \\ \text { Number of rows } & 8 & 8 & 8 & 8 \\ \text { Row width } & 1.25 \mathrm{~cm} & 0.5 \mathrm{~cm} & 1.25 \mathrm{~cm} & 1.25 \mathrm{~cm} \\ \text { Cube size } & 1.20 / 1.25 / 1.25 \mathrm{~cm} & 1.20 / 0.5 / 0.5 \mathrm{~cm} & 0.45 \mathrm{or} 1.20 / & 1.35 / 1.25 / \\ & & & 1.25 / 1.25 \mathrm{~cm} & 1.25 \mathrm{~cm}\end{array}$

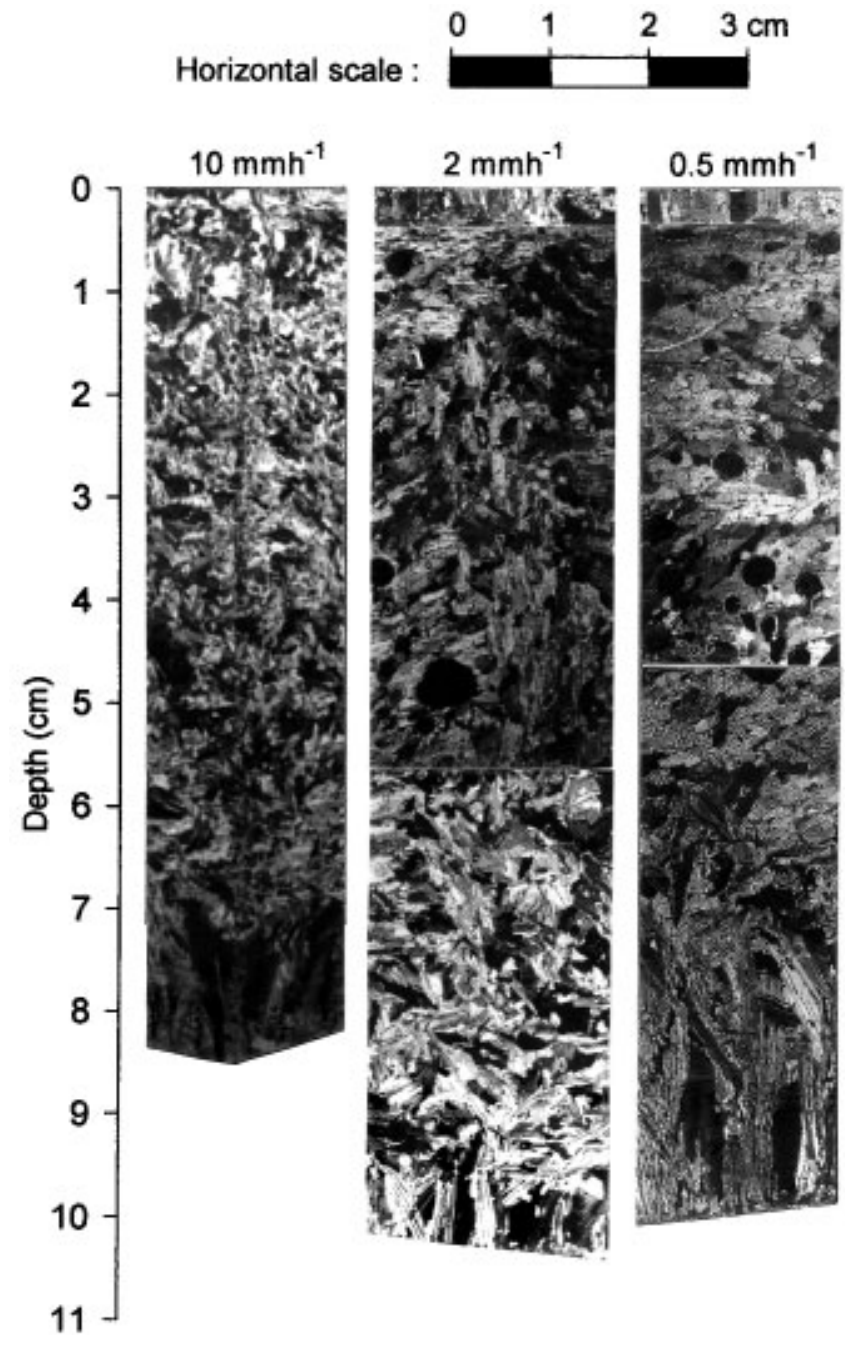

Fig. 4. Vertical thin-section photographs between crossed polarizers from the three experimental blocks. The bottom columnarcongelation layer showe the typical elongated vertical crystals with intra-crystalline brine-layer inclusions. In the granular ice, grain-size increases as growth rate decreases. A brine channel is clearly revealed in the centre of the photograph by the grain-size contrast in the $10 \mathrm{~mm} \mathrm{~h}^{-1}$ experiment, and by the vertical orientation of the disc-shaped crystals in the $2 \mathrm{~mm} \mathrm{~h}^{-1}$ experiment. In this latter case, careful examination of the orientation of the disc-shaped crystals suggests that these act as passive markers (during the early stages of consolidation) of the two upward branches of the convection cell that feed the downward movement in the brine channel. mimic the process of wind- and wave-induced frazil-ice formation, and the solution was regularly mixed to limit freezing on the sides of the container. After a few hours of production, the frazil crystals were separated from the solution with a $0.42 \mathrm{~mm}$ sieve, mixed with a new $\mathrm{NaCl} 34 \%$ solution (at the freezing point) and placed in the (lower) chamber of the freezing apparatus. The resulting "slush" (suspension of ice crystals in water) thickness was $8-10 \mathrm{~cm}$. The upper chamber of the apparatus was then fitted on the top of the lower one, so as to provide an intimate contact between the aluminium plate (Fig. 1) and the "slush".

Controlling the downward progression of the freezing front in the slush was not an easy task. A syringe needle was inserted delicately into the freezing medium through 17 sampling ports (Fig. 1) spaced at a resolution of $0.5 \mathrm{~cm}$ on the sides of the lower chamber. Depending on the degree of consolidation, the needle penetrated the ice mixture by a greater or lesser amount, as illustrated in Figure 2a for the $10 \mathrm{~mm} \mathrm{~h}^{-1}$ experiment. Results can then be plotted on graphs like those in Figure $2 \mathrm{~b}-\mathrm{d}$, where the two solid curves show needle penetration in the two extreme cases: the upper curve (black dots) indicates no penetration, while the lower curve (open circles) indicates the needle met no resistance. The behaviour of the upper curve confirms that the actual freezing front was reasonably close to the theoretical one (dotted lines in Fig. 2b-d).

On completion of the experiment, a $15 \mathrm{~cm} \times 20 \mathrm{~cm} \times$ thickness ice block was carefully removed from the central part of the lower chamber and stored at $-30^{\circ} \mathrm{C}$. Later, a central "core block" of $10 \mathrm{~cm} \times 10 \mathrm{~cm}$ section was sliced in plates, then rows and finally cubes of variable sizes, depending on the desired resolution (Table 1).

Given the limited volumes of samples (down to $0.13 \mathrm{~mL}$ ), it was unrealistic to measure salinity directly. Since highperformance liquid chromatography techniques allow us to work with much smaller sample volumes, we choose to use chlorinity as a proxy for the bulk salinity. Sample aliquots of $10 \mu \mathrm{L}$ were automatically diluted by a factor $10^{3}$ with tridistilled water (to avoid saturation of the column) and $\mathrm{Cl}^{-}$ measured using a Dionex DX-100 ion Chromatograph. Salinity was deduced from the chlorinity using a calibration curve based on 70 samples from a duplicate of the $10 \mathrm{~mm} \mathrm{~h}^{-1}$ experiment, where both chlorinity and salinity were measured. The linear regression obtained,

$$
S(\% 0)=1.805 \mathrm{Cl}^{-}(\%)+0.5\left(r^{2}=0.94\right),
$$

is very close to that used by Meese (1989). Given the sources of error inherent in the procedure, the precision of the salinity measurements from the chlorinity is only $\pm 1 \%$ (that is up to $10 \%$ of the measured values, for the lower range). However, we believe this provides a workable dataset since we are mainly interested in relative differences that will prove to be much larger than the maximum error of the estimates.

Before being sliced in individual rows, the horizontal plates were photographed under crossed polarizers (increasing the contrasts between ice and inclusions) to document the visual structures in the ice. Vertical plates were also reconstructed from their constitutive rows to produce a similar archive.

Finally, representative vertical thin sections were obtained from the remains of the initial ice block, using standard microtoming procedures (Langway, 1958). 
Mean salinity

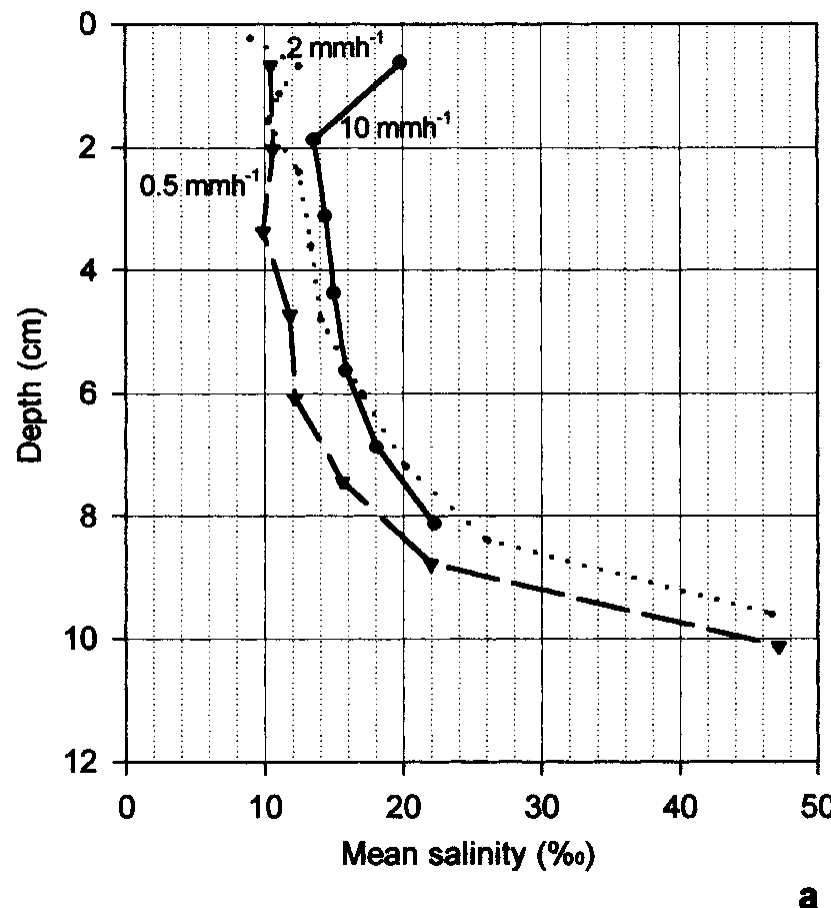

Standard deviation

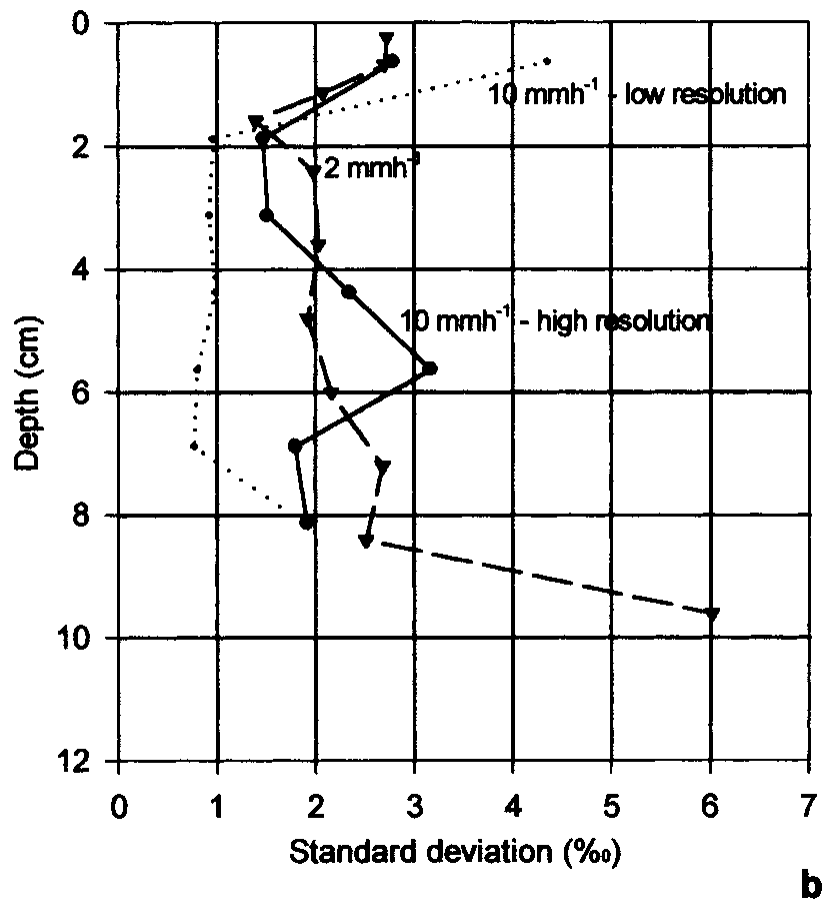

Fig. 5. Mean plate salinity as reconstructed from individual "cube" values in a given plate, and associated standard deviation.

\section{RESULTS AND DISCUSSION}

The chosen experimental procedure has some advantages and limitations that should be kept in mind when comparing the results to field measurements. Working at constant freezing rate in a given experiment increases our ability to tackle the initial conditions leading to channel growth. However, it does not adequately reproduce the long time evolution of the brine volume that would result from a decrease in icegrowth rate. Use of a finite reservoir allows better control of the variables but also results in progressive salinization of the water, which is ultimately reflected in the bulk ice salinity. Finally, by using a simpler two-step approach ((a) growth of frazil and (b) consolidation of a whole slush layer) we reproduce an "internal" phase boundary process which only partially covers the field phenomenon, where both steps could sometimes occur simultaneously.

\section{Growth-rate control on ice textures and brine-channel development}

Figures 3 and 4 show vertical thick and thin sections, respectively, from the three experimental blocks. It is obvious from Figure 3 that brine channels are well developed in the consolidated granular ice, for both 10 and $2 \mathrm{~mm} \mathrm{~h}^{-1}$. No channels appear at the lowest growth rate, although it should be mentioned that one single channel was counted outside of the limits of the sampled block. Details of the channel's geometry are also visible on the photographs of Figure 6 (shown later), both along the vertical (Fig. 6e-h) and in selected horizontal plates (Fig. 6a-d). The channels are fairly rectilinear and typically fed by lateral channels, as described in natural and experimental congelation sea ice (Bennington, 1967; Lake and Lewis, 1970; Eide and Martin, 1975; Wakatsuchi and Kawamura, 1987; Kawamura, 1988; Weissenberger and others, 1992; Cottier, 1999; Cottier and others, 1999). The central channel is $1.2-1.5 \mathrm{~mm}$ in diameter. The channels are more numerous at higher growth rates, but their lateral extension is reduced when compared to lower growth rates. Lateral channels, generally considered as "feeder" tubes of the main central channel (i.e. Lake and Lewis, 1970; Cottier, 1999; Cottier and others, 1999), are shorter but more numerous at higher growth rates. These observations can be viewed as the result of the reduced connectivity at lower ice temperatures (e.g. Niedrauer and Martin, 1979), which would indeed be the case for higher growth rates. Nearly all the tributary channels run downward with a mean angle of $40-56^{\circ}$ to the vertical. This range is in agreement with that described by Lake and Lewis (1970) and Criminale and Lelong (1984). It should, however, be noted that a few tributaries run upwards, towards the main channel (middle left of the photograph in Fig. 6g). In some cases, the main channel suffers a clear disruption before resuming in the same alignment.

Figure 4 shows the textural characteristics of the granular ice for the three experiments. In all cases, there is a clear textural change at the bottom of the section, where a transition takes place to elongated vertical crystals with internal brine layers, typical of congelation-ice growth. Whilst the initial grain-size in the slush was sub-millimetric for all experiments, the grain-size clearly depends on the growth rate in the consolidated ice. A marked difference to the textures which usually result from the consolidation of wind- and wave-induced frazil in Nature is the "disc" appearance of the grains. This probably results from a "geometrical effect", caused by the reduction of mechanical interference between grains during the freezing of frazil ice in a confined environment. Subsequent growth during the consolidation process would have preserved the disc shape of the crystals. Careful examination of Figure $4 \mathrm{a}$ and $\mathrm{b}$ reveals the signature of the brine channels on the texture. In the fast $10 \mathrm{~mm} \mathrm{~h}^{-1}$ growth experiment, the channel is characterized by a very fine-grained matrix, partially resulting from rapid freezing during storage of the 
$10 \mathrm{mmh}^{-1}$ - low resolution

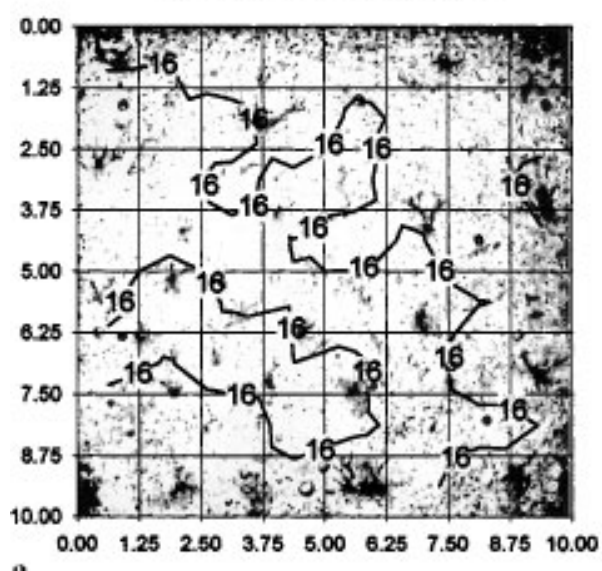
a

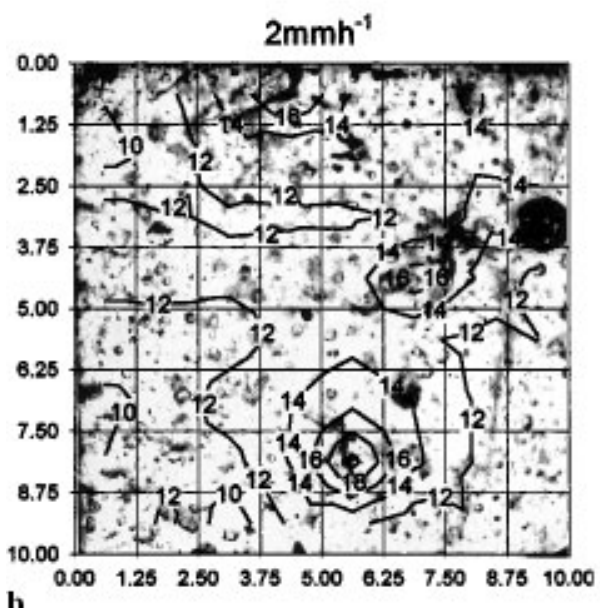

b

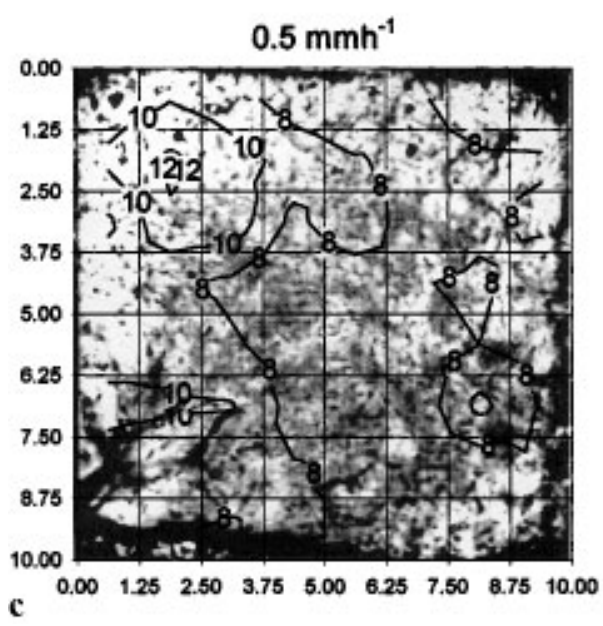

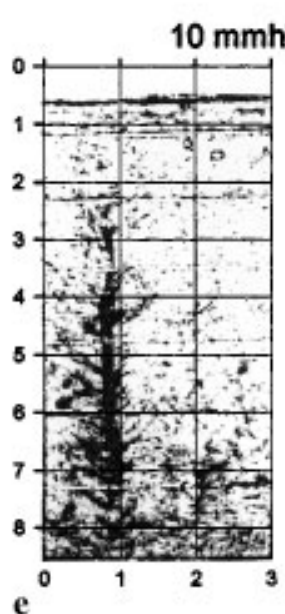
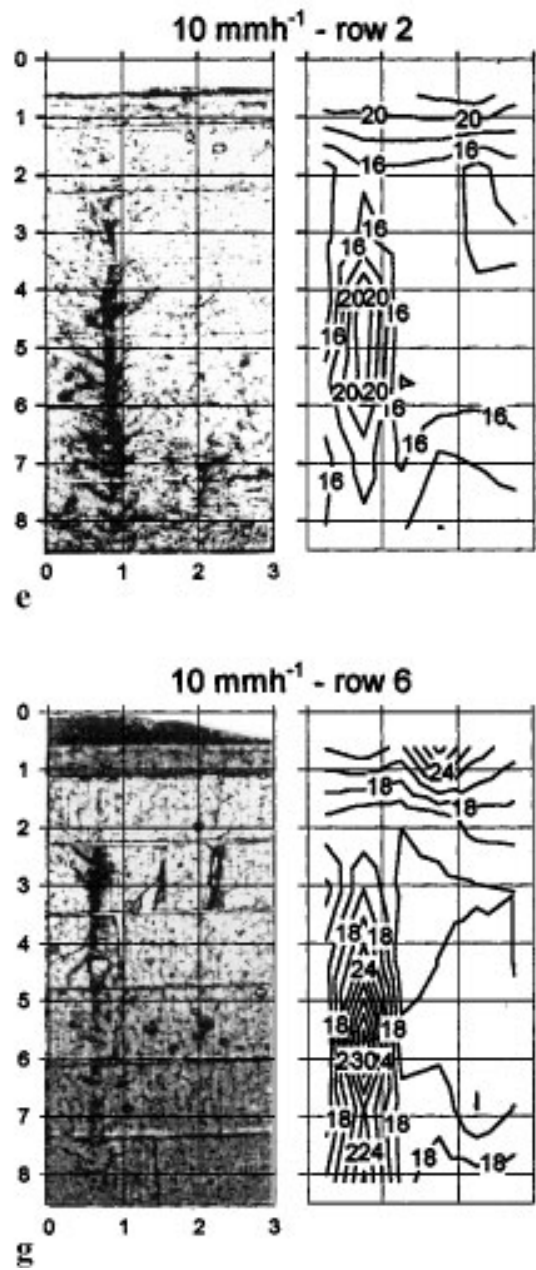

$10 \mathrm{mmh}^{-1}$ - high resolution

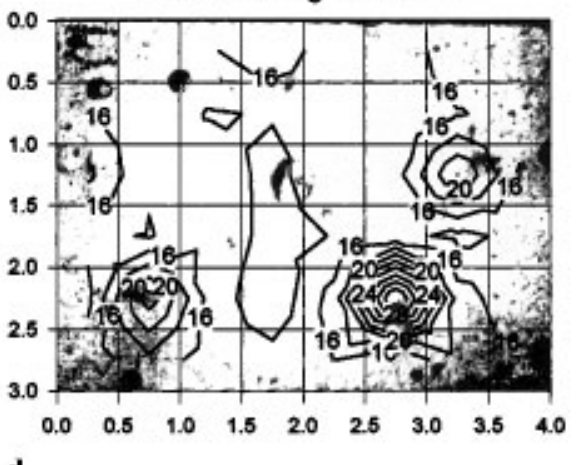

d

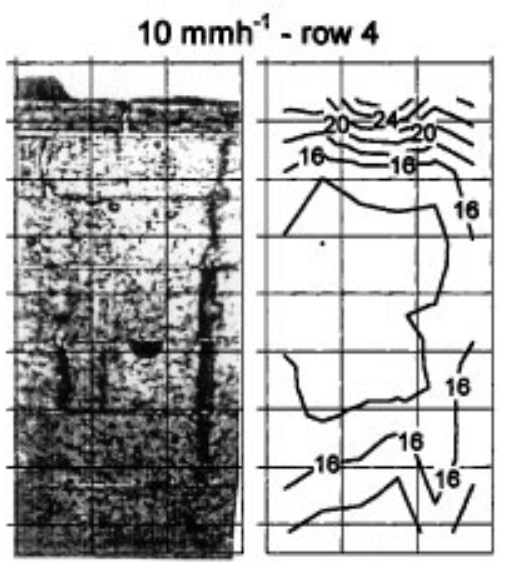

f

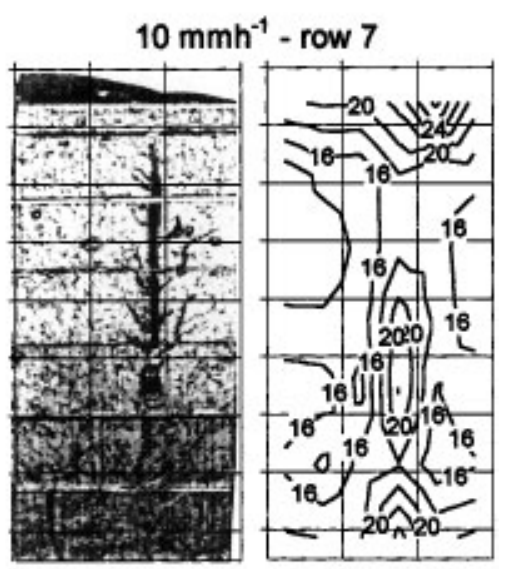

h

Fig. 6. Selected two-dimensional salinity diagrams in horizontal $(a-d)$ and vertical $(e-h)$ sections for the three experiments. Isohaline curves are in \%o. In the horizontal sections, gridlines (scale in centimeters) delineate individual cube sides (see Table 1 for details). In the vertical sections, gridlines correspond to depth and horizontal scales in centimetres. For ease of interpretation, isohaline maps have been separated from the equivalent transmitted-light picture in the vertical sections.

sample at $-30^{\circ} \mathrm{C}$. At a slower growth rate, there is no contrast in crystal size between the channel and its surroundings, but the downward flow of brine in the channel has clearly reoriented the discs in a sub-vertical position. Textures on both sides of the channel also suggest (particularly on the top righthand side of Fig. 4b) that a convection process similar to that described by Niedrauer and Martin (1979) for the skeletal layer of congelation ice occurs in granular ice. The difference here is that the geometry of the slush offers less resistance to the upward fluxes. This flux has a lateral component which feeds into the main channel.

\section{Mean salinity profiles}

For the three experiments, Figure 5a shows the mean salinity profile, reconstructed from the salinity of each individual cube sample. The $10 \mathrm{~mm} \mathrm{~h}^{-1}$ experiment shows the typical C-shaped profile found in both natural and experimental 

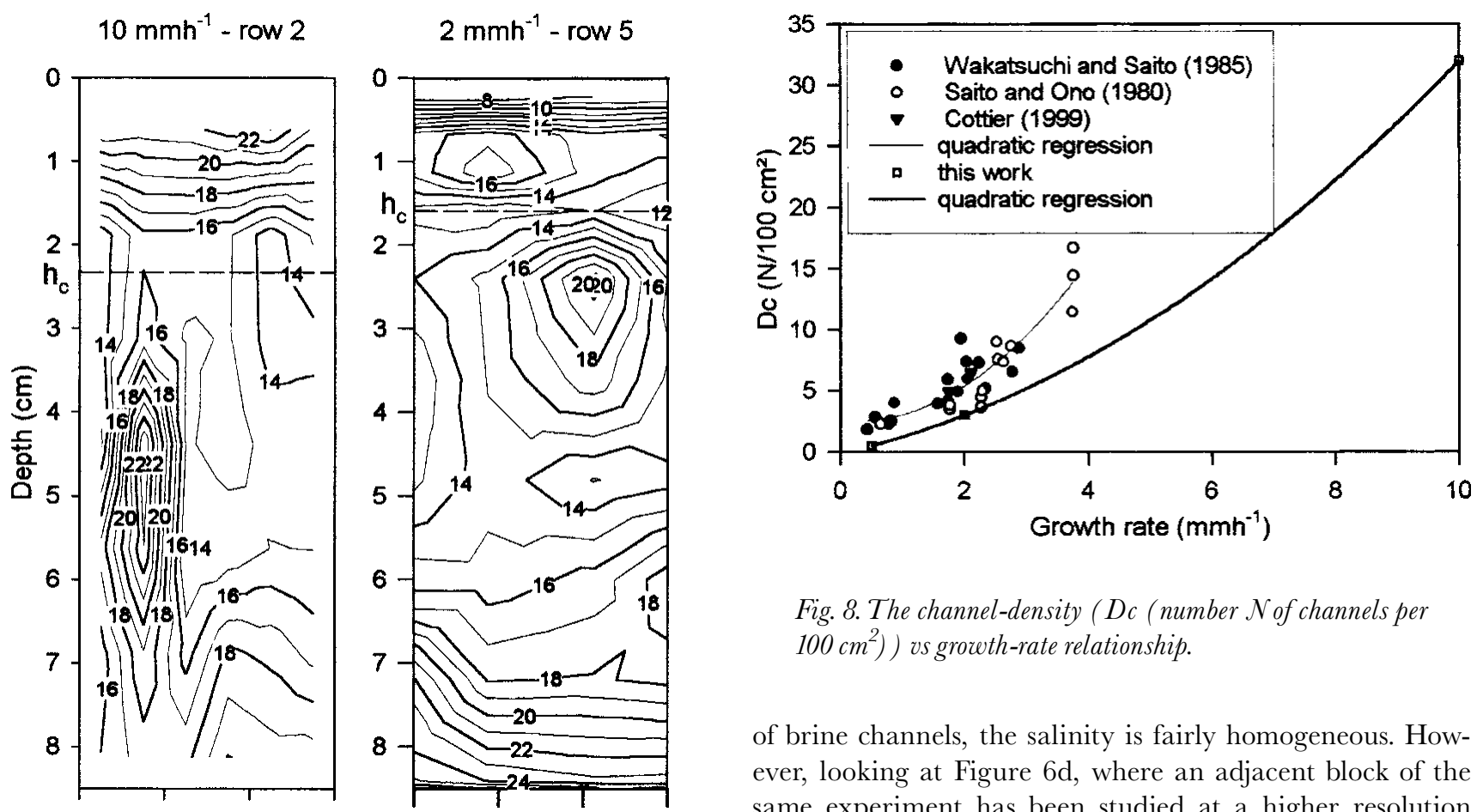

Fig. 7. Bulk salinity distribution in a vertical section along channels for the 10 and $2 \mathrm{~mm} \mathrm{~h}^{-1}$ experiment. The critical depth $\left(h_{\mathrm{c}}\right)$ for the onset of internally driven convection that is necessary to initiate channel development is smaller at lower freezing rates, indicating the dominating effect of porosity over concentration gradients (see text for details).

columnar sea ice (i.e. Nakawo and Sinha, 1981; Cottier and others, 1999). The 2 and $0.5 \mathrm{~mm} \mathrm{~h}^{-1}$ experiments do not show the surface enrichment generally attributed to upward brine expulsion during cooling of the surface layers. This could be due to the experimental upper boundary conditions which obviously differ from an atmospheric boundary.

The small depth of the reservoir causes evolution towards a closed system rather early in the freezing process. Therefore, the salinity in the ice reflects the increase of the salt concentration in the water reservoir as freezing progresses. The very high salinities reached at the bottom of the reservoir at the end of the process, coupled with the increased porosity of the bottom ice layers, explain the enhancement of the lower curvature of the $\mathrm{C}$-shaped profile in comparison with natural sea ice (e.g. maximum 25\% in Eclipse Sound, Baffin Island (Nakawo and Sinha, 1981)). Intercomparison of the three profiles supports the view that decreasing the growth rate increases the efficiency of salt rejection, thereby depleting the upper layers and enriching the lower ones in this closed system.

\section{Chemical signature of brine channels}

Details of the salinity distribution in the blocks are shown in specific horizontal or vertical sections in Figure 6. The discrete salinity values from individual cubes have been interpolated to produce isohaline curves. These are compared to black-and-white photography showing the visual features in the same area.

A striking feature of Figure 6a is the apparent contradiction between the visual image of the horizontal plate (4.8$6 \mathrm{~cm}$ depth interval) in the $10 \mathrm{~mm} \mathrm{~h}^{-1}$ experiment, and the corresponding salinity map. Despite the occurrence of a large number of dark spots on the image, suggesting a high density

\section{Fig. 8. The channel-density ( Dc (number $\mathcal{N}$ of channels per $\left.100 \mathrm{~cm}^{2}\right)$ ) vs growth-rate relationship.}

of brine channels, the salinity is fairly homogeneous. However, looking at Figure 6d, where an adjacent block of the same experiment has been studied at a higher resolution (Table 1), the correspondence between visual channel crosssections and salinity maxima becomes clear. This demonstrates the importance of the choice of the sampling resolution for the reliability of the results. Cottier and others (1999) argue that the choice of a $20 \mathrm{~mm}$ sampling grid is justified as the optimum sampling resolution for the study of brine channels in young sea ice. The example above suggests that this is only true for a typical range of sea-ice growth rates. Figure $6 \mathrm{~b}$ shows traces of two to three brine channels, formed at $2 \mathrm{~mm} \mathrm{~h}^{-1}$ growth rate, that also clearly correspond to salinity maxima. The salinity maxima are, however, lower (16-18\%o vs $22-28 \%$ ), and the gradients smaller $\left(4-8 \% \mathrm{~cm}^{-1}\right.$ vs $13-20 \% \mathrm{~cm}^{-1}$ ), than at $10 \mathrm{~mm} \mathrm{~h}^{-1}$. Similarly, the gradients observed at $2 \mathrm{~mm} \mathrm{~h}^{-1}$ are generally higher than those of maximum $5.2 \mathrm{psu}$ (practical salinity units) $\mathrm{cm}^{-1}$ mentioned by Cottier (1999), for columnar-congelation ice grown at rates in the range $1.2-0.5 \mathrm{~mm} \mathrm{~h}^{-1}$. Another way to detect this spatial variability is to look at the standard deviation of the mean salinity values plotted in Figure 5b (discarding the upper and lower boundary plates, where brine upward expulsion and reservoir "close-off", respectively, are likely to have altered the distribution). The low-resolution $10 \mathrm{~mm} \mathrm{~h}^{-1}$ experiment gives a $\sigma$-value between $\pm 0.77 \%$ ond $\pm 0.99 \%$, whilst in the high-resolution $10 \mathrm{~mm} \mathrm{~h}^{-1}$ experiment $\sigma$ fluctuates between $\pm 1.47 \%$ and $\pm 3.16 \%$. In the $2 \mathrm{~mm} \mathrm{~h}^{-1}$ experiment, the range is $\pm 1.39 \%$ o to $\pm 2.68 \%$. The maximum salinity difference between adjacent cubes is $16.04 \%$ o for the $10 \mathrm{~mm} \mathrm{~h}^{-1}$ experiment and $7.84 \%$ for the $2 \mathrm{~mm} \mathrm{~h}^{-1}$ experiment. In the slowest $0.5 \mathrm{~mm} \mathrm{~h}^{-1}$ experiment, no correlation exists between visual observations of impurities and salinity. Slight gradients exist, but they are much lower than those described in the faster experiments.

The correspondence between brine-channel traces and salinity maxima exists not only in horizontal sections, but also along the vertical, thereby indicating that we are dealing with channels, and not pockets of brine. This is clearly illustrated in specific sections of the high-resolution $10 \mathrm{~mm} \mathrm{~h}^{-1}$ block. Figure $6 \mathrm{e}^{-} \mathrm{h}$, respectively, represent rows 2, 4, 6 and 7 of Figure $6 d$, but for the whole depth range. Not only are the channels well delineated by high-salinity areas with strong gradients surrounded by areas of uniform salinity, but also 
the channel disruptions described in a previous subsection are found to be "saddles" of minimum salinity. These disruptions might have developed during low-temperature $\left(-30^{\circ} \mathrm{C}\right)$ storage before the samples were processed.

It is also interesting to note that the salinity maxima in the upper $2 \mathrm{~cm}$ (especially Fig. $6 \mathrm{f}-\mathrm{h}$ ) are offset with regard to the channel's location, suggesting a particular initiation process. Brine-channel features only start to develop below a depth of a few centimetres. This critical depth, below which convection begins, is achieved when the driving buoyancy of the enriched brine in the porous medium overcomes the resistance of the solid matrix. As defined by Wettlaufer and others (1997a, equation 1), internally driven convection in the form of brine channels will begin once the porous-medium Rayleigh number is exceeded. The critical thickness for brine-channel initiation $h_{\mathrm{c}}$ can be expressed as:

$$
h_{\mathrm{c}}=\frac{\operatorname{Ra} \kappa \nu}{g \beta \Delta C \Pi(\Phi)},
$$

where $\mathrm{Ra}$ is the porous medium Rayleigh number, $g$ is the acceleration due to gravity, $\beta \Delta C$ is the difference in the liquid density across the mushy layer, $\kappa$ and $\nu$ are the thermal diffusivity and kinematic viscosity of the liquid and $\Pi$ is the permeability of the layer, which is a function of the solid fraction $\Phi$. At a reduced growth rate, the thermal driving is smaller, the resultant solid fraction of the mushy layer smaller, and the permeability higher. Although the difference in the liquid density across the mushy layer should be lower, supposing that thermodynamic equilibrium is achieved, the net effect observed by Wettlaufer and others (1997a) is a more rapid development of the brine-channel system $\left(h_{\mathrm{c}}\right.$ lower). This is also the case here, if we compare the 10 and $2 \mathrm{~mm} \mathrm{~h}^{-1}$ experiments in vertical sections (Fig. 7). If, however, the growth rate is further reduced, purely diffusional processes will become dominant and will lower the liquid-density difference across the mushy layer sufficiently to reduce or even prevent brinechannel development. This process might be responsible for interruption of channels in natural sea ice. A similar rationale would explain the apparent lack of convection features in what is often referred to as "marine ice" that results from consolidation of loose frazil at the bottom of ice shelves in conditions of extremely slow growth.

\section{Growth-rate control on brine-channel density}

Wakatsuchi and Saito (1985) have compared brine-channel densits in columnar-congelation sea ice from various studies (including Saito and Ono, 1980) and shown a noticeable dependence on the initial freezing rate (white and black dots in Fig. 8). Cottier's (1999) experimental values fit with the previous set (black triangles in Fig. 8). It is worth comparing these observations with the experimental results on brine-channel development in columnar sea ice from Wettlaufer and others (1997b). For a given initial water salinity, these authors demonstrate (their fig. 17) that once internally driven convection has begun (full development of brine channels), the mean solid fraction is identically dependent on depth in all experiments, independent of the initial thermal driving. If the Wakatsuchi and Saito (1985) relationship holds, this means that equivalent porosities are achieved with different channel configurations, these configurations depending on the initial freezing conditions. Thus for higher initial growth rates, the reduced porosity (connectivity) at the onset of convection favours a larger number of smaller channels and vice versa.
Our dataset in granular sea ice confirms the general trend of an increase in channel density with the growth rate. However, although it relies on a limited number of experiments, these provide lower channel densities at equivalent rates. We suggest that this is due to a more efficient transport process in a loose granular matrix, as opposed to the more constraining network of the brine layers/ice-crystal lamellae of the skeletal layer in columnar ice.

\section{GONGLUSIONS}

The nature of the present high-resolution study, involving more than 2000 chlorinity/salinity measurements, results in a limited number of experiments. However, the large range of freezing rates addressed and the consistency of the results allow us to obtain a number of important findings.

Brine-channel development in experimental granular sea ice proves to be as important as in columnar-congelation ice. This is consistent with the "mushy-layer" concept developed by Grae Worster (1992a, b) for solidification of alloys and invoked by Wettlaufer and others (1997a, b) for columnar sea-ice formation, in which brine channels develop as convection features once a critical depth is reached at the onset of freezing. Intuitively, a granular medium like the slush formed by loose frazil-ice crystals in sea water should be even closer to the "mushy-layer" structure than the "skeletal layer" of columnar-congelation ice. The reduced geometrical constraints on the transport of brine probably enhance the efficiency of each individual channel and therefore lower the channel density with respect to those found in columnar ice (Wakatsuchi and Kawamura, 1987). On the other hand, our experiments are similar to those in columnar ice, in that the number of brine channels decreases drastically with the growth rate (Fig. 8). This probably reflects the dependence of the brine-channel distribution on porosity (and therefore connectivity) at the onset of internally driven convection. Comparing the critical depth $\left(h_{\mathrm{c}}\right)$ for brine-channel initiation at different growth rates in our granular-ice experiments confirms the dominant effect of porosity $(\Pi(\Phi)$ in Equation (2)) over concentration (density) gradients $(\beta \Delta C$ in Equation (2)) across the mushy layer in driving internal convection by exceeding the porous-medium Rayleigh number (Wettlaufer and others, 1997a, b). However, the absence of brine channels in our lower-growth-rate experiment $\left(0.5 \mathrm{~mm} \mathrm{~h}^{-1}\right)$ suggests the existence of another regime where diffusional processes become dominant in the boundary layer at the ice/slush interface $\left(\Delta C\right.$ tends to 0 and $h_{\mathrm{c}}$ tends to $\infty$ in Equation (2)). Switching between the two regimes might be responsible for the interruption and re-initiation of channels in natural sea ice. Predominance of this alternative regime at extremely slow growth rates would explain the lack of convection features in "marine ice" which forms at the bottom of ice shelves.

Future work should further document the "initialgrowth-rate/channel-density" relationship in granular ice. It should also aim to validate the hypothesis that geometrical constraints (connectivity) control (a) the dependence of channel density on the initial growth rate in both granular and columnar ice, and (b) the offset of that relationship between the two different ice types. Experiments allowing controlled growth at extremely low freezing rates should also be valuable in detecting an alternative diffusion-con- 
trolled regime, in which internally driven convection would be prevented.

\section{AGKNOWLEDGEMENTS}

The authors wish to thank two anonymous reviewers, T. Kawamura and P. Langhorne for considerably improving the quality of the manuscript. This project has benefited from the support of the Belgian Antarctic Program (SSTC, Science Policy Office). J.L.T. is Research Associate at the Fonds National de la Recherche Scientifique (FNRS), Belgium.

\section{REFERENGES}

Bennington, K. O. 1967. Desalination features in natural sea ice. F. Glaciol., 6(48), 845-857.

Cottier, F. R. 1999. Brine distribution in young sea ice. (Ph.D. thesis, University of Cambridge.)

Cottier, F. R., H. Eicken and P. Wadhams. 1999. Linkages between salinity and brine channel distribution in young sea ice. F. Geophys. Res., 104(C7), $15,859-15,871$.

Cox, G. F. N. and W. F. Weeks. 1988. Numerical simulations of the profile properties of undeformed first-year sea ice during the growth season. 7. Geophys. Res., 93 (C10), 12,449-12,460.

Criminale, W. O., Jr and M.-P. Lelong. 1984. Optimum expulsion of brine from sea ice. 7. Geophys. Res., 89(C3), 3581-3585.

Eicken, H. 1998. Deriving modes and rates of ice growth in the Weddell Sea from microstructural, salinity and stable-isotope data. In Jeffries, M. O., ed. Antarctic sea ice: physical processes, interactions and variability. Washington, DC, American Geophysical Union, 89-122. (Antarctic Research Series 74.)

Eide, L. I. and S. Martin. 1975. The formation of brine drainage features in young sea ice. F. Glaciol., 14(70), 137-154.
Grae Worster, M. 1992a. The dynamics of mushy layers. In Davis, H. E. H. S. H., U. Müller and M. Grae Worster, eds. Interactive dynamics of convection and solidification. Dordrecht, etc., Kluwer, 113-138.

Grae Worster, M. 1992b. Instabilities of the liquid and mushy regions during solidification of alloys. 7. Fluid Mech., 237, 649-669.

Kawamura, T. 1988. Experimental study on distribution of brine drainage channels in sea ice. In Saeki, H. and K.-i Hirayama, eds. IAHR88, 9th IAHR International Symposium on Ice, 23-27 August 1988, Sapporo, Japan. Proceedings. Sapporo, International Association for Hydraulic Research. Committee on Ice Problems, 219-228.

Lake, R. A. and E. L. Lewis. 1970. Salt rejection by sea ice during growth. $\mathcal{F}$. Geophys. Res., 75 (3), 583-597.

Langway, C. C., Jr. 1958. Ice fabrics and the universal stage. SIPRE Tech. Rep. 62.

Meese, D. A. 1989. The chemical and structural properties of sea ice in the southern Beaufort Sea. CRREL Rep. 89-25.

Nakawo, M. and N. K. Sinha. 1981. Growth rate and salinity profile of firstyear sea ice in the High Arctic. 7. Glaciol., 27(96), 315-330.

Niedrauer, T. M. and S. Martin. 1979. An experimental study of brine drainage and convection in young sea ice. 7. Geophys. Res., 84(C3), 1176-1186.

Saito, T. and N. Ono. 1980. Percolation of sea ice. II. Brine drainage channels in young sea ice. Low Temp. Sci., Ser. A, 39, 127-132.

Wakatsuchi, M. and T. Kawamura. 1987. Formation processes of brine drainage channels in sea ice. f. Geophys. Res., 92(C7), 7195-7197.

Wakatsuchi, M. and T. Saito. 1985. On brine drainage channels of young sea ice. Ann. Glaciol., 6, 200-202.

Weissenberger, J., G. Dieckmann, R. Gradinger and M. Spindler. 1992. Sea ice: a cast technique to examine and analyze brine pockets and channel structure. Limnol. Oceanogr., 37(1), 179-183.

Wettlaufer, J. S., M. Grae Worster and H. E. Huppert. 1997a. Natural convection during solidification of an alloy from above with application to the evolution of sea ice. F. Fluid Mech., 344, 291-316.

Wettlaufer, J. S., M. Grae Worster and H. E. Huppert. 1997b. The phase evolution of young sea ice. Geophys. Res. Lett., 24(10), 1251-1254. 